\title{
Pilot-Symbol-Assisted Channel Estimation for Space-Time Coded OFDM Systems
}

\author{
King F. Lee \\ Multimedia Architecture Lab, Motorola Labs, Schaumburg, IL 60196, USA \\ Email: King.Lee@motorola.com \\ Douglas B. Williams \\ School of Electrical and Computer Engineering, Georgia Institute of Technology, Atlanta, GA 30332, USA \\ Email: douglas.williams@ece.gatech.edu
}

Received 31 May 2001 and in revised form 5 March 2002

\begin{abstract}
Space-time coded orthogonal frequency division multiplexing (OFDM) transmitter diversity techniques have been shown to provide an efficient means of achieving near optimal diversity gain in frequency-selective fading channels. For these systems, knowledge of the channel parameters is required at the receivers for diversity combining and decoding. In this paper, we propose a low complexity, bandwidth efficient, pilot-symbol-assisted (PSA) channel estimator for multiple transmitter OFDM systems. The pilot symbols are constructed to be nonoverlapping in frequency to allow simultaneous sounding of the multiple channels. The timevarying channel responses are tracked by interpolating a set of estimates obtained through periodically transmitted pilot symbols. Simulations are used to verify the effectiveness of the proposed estimator and to examine its limitations. It is also shown that the PSA channel estimator has a lower computational complexity and better performance than a previously proposed decisiondirected minimum mean square error MMSE channel estimator for OFDM transmitter diversity systems.
\end{abstract}

Keywords and phrases: transmitter diversity, OFDM, channel estimation, pilot symbols, interpolation.

\section{INTRODUCTION}

The mobile wireless channel suffers from multipath fading that severely attenuates the received signal during periods of deep fades. Spatial diversity is a well-known technique for improving the performance and reliability of wireless communications over fading channels. Traditionally, spatial diversity has been implemented at the receiver end by using multiple antennas at the receiver and then combining signals to improve the quality of the received signal. Unfortunately, receiver diversity requires multiple, widely-spaced antennas and multiple radio frequency (RF) front-end circuits at the receiver. This multiplicity of receiver front-end hardware is undesirable and impractical for portable receivers, such as pagers or cellular handsets, where physical size and current drain are important constraints. Transmitter diversity, on the other hand, can be implemented with multiple spatially separated antennas at the transmitter and requires only a single antenna and front-end circuit at the receiver. Transmitter diversity techniques are, therefore, very suitable for paging, cellular, and portable wireless data services, where a small number of base stations serve a large number of mobile users and where spatially separated antennas can be easily implemented at the base stations. Hence, transmitter diversity has received strong interest in recent years, especially in the mobile communications research community. Furthermore, the channels over which these high data rate mobile communications systems operate are generally frequency-selective, so transmitter diversity techniques that are effective in frequency-selective fading channels are of special interest.

A number of space-time coded orthogonal frequency division multiplexing (OFDM) transmitter diversity techniques have recently been proposed for frequency-selective fading channels $[1,2,3,4]$. These techniques are capable of achieving near optimal diversity gain when the receivers have perfect knowledge of the channels. In practice, the channel parameters have to be estimated at the receivers. Channel estimation techniques for conventional OFDM systems have been studied extensively by many researchers $[5,6,7$, $8,9,10,11]$. However, channel estimation for OFDM systems with transmitter diversity has seen only limited development so far. Channel estimation for transmitter diversity systems is complicated by the fact that signals transmitted simultaneously from multiple antennas become interference for each other during the channel estimation process. In [12], a decision-directed minimum mean square error (MMSE) channel estimator for OFDM transmitter diversity systems 


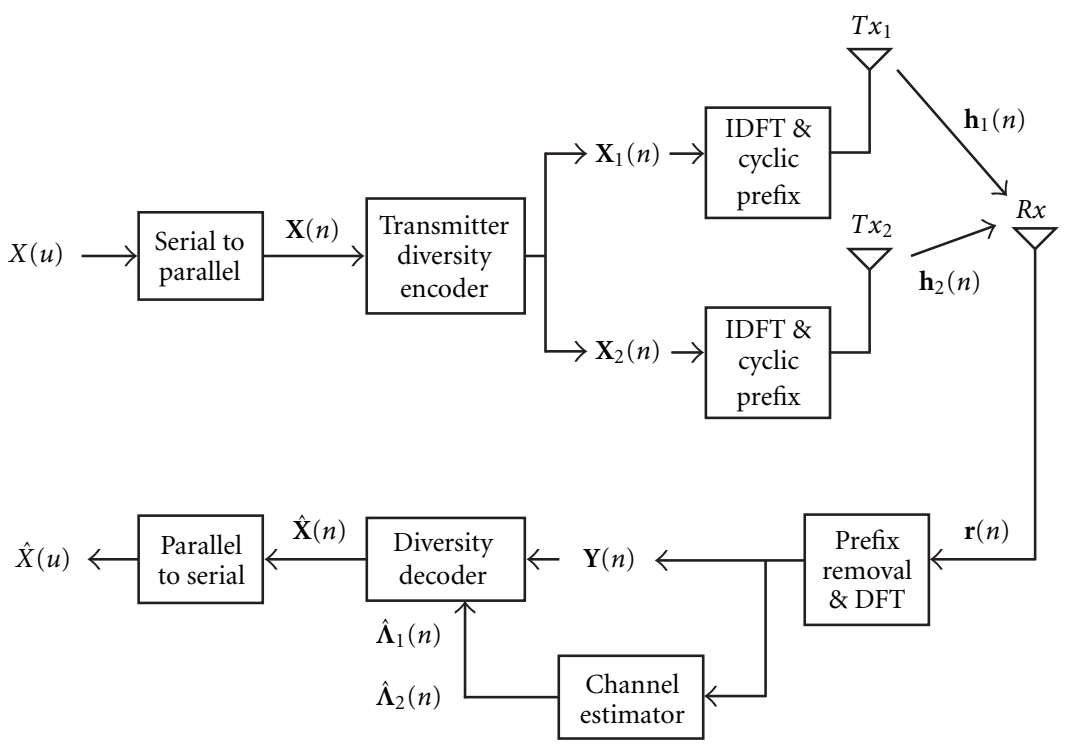

FIGURE 1: Block diagram of a two-branch OFDM transmitter diversity system.

was proposed. The primary shortcoming of the MMSE channel estimation approach is the high computational complexity required to update the channel estimates during the data transmission mode. In this paper, we investigate a low complexity channel estimation technique for multiple transmitter OFDM systems. The proposed technique uses bandwidth efficient pilot symbols to facilitate temporal estimation of the multiple channel responses. Simple interpolation filters are then used to update the estimates during the data transmission mode.

\section{OFDM TRANSMITTER DIVERSITY SYSTEMS}

A block diagram of a general two-branch OFDM transmitter diversity system is shown in Figure 1. Let $X(u)$ denote the input serial data symbols with symbol duration $T_{S}$. The serial to parallel converter collects $K$ serial data symbols into a data vector $\mathbf{X}(n)=[X(n, 0) X(n, 1) \cdots X(n, K-1)]^{T}$, which has a block duration of $K T_{S} .{ }^{1}$ The transmitter diversity encoder codes $\mathbf{X}(n)$ into two vectors $\mathbf{X}_{1}(n)$ and $\mathbf{X}_{2}(n)$ according to an appropriate coding scheme as in $[1,2,3,4]$. The coded vector $\mathbf{X}_{1}(n)$ is modulated by an inverse discrete Fourier transform (IDFT) into an OFDM symbol sequence. A length $G$ cyclic extension is added to the OFDM symbol sequence, and the resulting signal is transmitted from the first transmit antenna. Similarly, the vector $\mathbf{X}_{2}(n)$ is modulated by an IDFT, cyclically extended, and transmitted from the second transmit antenna. Let $\mathbf{h}_{1}(n)$ denote the impulse response of the channel between the first transmit antenna and the receiver and $\mathbf{h}_{2}(n)$ denote the impulse response of the channel between the second transmit antenna and the receiver. The length of the cyclic extension is chosen to be greater than

\footnotetext{
${ }^{1}$ Throughout the paper we use the notation that $A(n, k)$ is the $k$ th element of the vector $\mathbf{A}(n)$.
}

or equal to $L$, the order of the channel impulse responses, that is, $G \geq L$. At the receiver, the received signal vector first has the cyclic prefix removed and is then demodulated by a discrete Fourier transform (DFT) to yield the demodulated signal vector $\mathbf{Y}(n)$. Assuming that the channel impulse responses remain constant during the entire block interval, it can be easily shown that the demodulated signal is given by

$$
\mathbf{Y}(n)=\boldsymbol{\Lambda}_{1}(n) \mathbf{X}_{1}(n)+\boldsymbol{\Lambda}_{2}(n) \mathbf{X}_{2}(n)+\mathbf{Z}(n),
$$

where $\boldsymbol{\Lambda}_{1}(n)$ and $\boldsymbol{\Lambda}_{2}(n)$ are two diagonal matrices whose elements are the DFTs of the respective channel impulse responses, $\mathbf{h}_{1}(n)$ and $\mathbf{h}_{2}(n)$, and $\mathbf{Z}(n)$ is the DFT of the channel noise. Elements of $\mathbf{Z}(n)$ are generally assumed to be additive white Gaussian noise with variance $\sigma_{Z}^{2}$. Clearly, the demodulated signal vector $\mathbf{Y}(n)$ is the superposition of the two encoded vectors $\mathbf{X}_{1}(n)$ and $\mathbf{X}_{2}(n)$, which makes estimation of the channel parameters (i.e., $\mathbf{h}_{1}(n)$ and $\mathbf{h}_{2}(n)$ or, equivalently, $\boldsymbol{\Lambda}_{1}(n)$ and $\left.\boldsymbol{\Lambda}_{2}(n)\right)$ from $\mathbf{Y}(n)$ challenging for transmitter diversity systems, especially during the data transmission mode.

\section{CHANNEL ESTIMATION FOR OFDM TRANSMITTER DIVERSITY SYSTEMS}

There are two common strategies for estimating the parameters of fading channels: decision-directed channel estimation and pilot-symbol-assisted (PSA) channel estimation. With decision-directed channel estimation, decoded symbols $\hat{\mathbf{X}}(n)$ at the output of the decision device, or more frequently after the error-correction decoder, are used for estimating the channel parameters during the data transmission mode. Since past decisions are used to estimate the channel parameters, decision-directed channel estimation is susceptible to error propagation, especially during a deep fade. Therefore, 
even with decision-directed channel estimation, known symbols ${ }^{2}$ are periodically transmitted to avoid excessive error propagation. The channel estimator in [12] is essentially a decision-directed channel estimator where the decoded data symbols are used, during the data transmission mode, to estimate the set of channel parameters that minimizes the meansquare error (MSE) cost function

$$
\sum_{k=0}^{K-1}\left|Y(n, k)-\sum_{m=1}^{M} \Lambda_{m}(n, k) X_{m}(n, k)\right|^{2},
$$

where $M$ is the number of transmitters. With PSA channel estimation, known pilot symbols are inserted into the transmit symbol stream, usually at a regular interval. At the receiver, the pilot symbols are extracted to provide a temporal estimate of the channel parameters at the pilot instants. These temporal estimates are then either filtered or interpolated to provide estimates of the channel parameters during the data transmission mode.

It is interesting to note that although both the decisiondirected channel estimator and the PSA channel estimator estimate the channel parameters using known symbols in the form of training and pilot symbols, there is a major difference between the two estimators during the data transmission mode. With the decision-directed approach, decoded symbols are used to update the channel estimates continuously during the data transmission mode. On the other hand, with the PSA approach, decoded symbols during the data transmission mode are not used to determine the channel. Channel estimates are generated either by filtering or interpolating the temporal estimates obtained at the pilot instants. This difference has special significance for transmitter diversity systems where the multiple transmitted signals tend to interfere with the channel estimation process. During the training or pilot mode, the interferences among the multiple transmitted signals can be easily minimized for the decision-directed channel estimator or PSA channel estimator by employing properly designed orthogonal training symbols $[12,13]$ or pilot symbols. However, there is no such "luxury" during the data transmission mode, because the multiple transmitted signals typically correspond to randomly distributed data symbols. Hence, the MMSE solution for finding the "best" estimate amid the interfering signals, such as in [12], is indeed the logical approach for decisiondirected channel estimation for transmitter diversity systems. For the PSA channel estimator, however, the main challenges are in minimizing the interferences among the pilot symbols from the multiple transmitters and in the design of the interpolator. The interferences during the data transmission mode, which are more difficult to resolve, are not a concern at all for PSA channel estimators. Consequently, PSA channel estimation will be shown to be the better choice for transmitter diversity systems.

\footnotetext{
${ }^{2}$ To avoid possible confusion with PSA channel estimation approaches, these known symbols for decision-directed channel estimation are sometimes referred to as training symbols.
}

\subsection{Pilot symbols for multiple transmitter OFDM systems}

Pilot-symbol-assisted (PSA) channel estimation techniques for single transmitter systems have been proposed and are well understood $[14,15]$. However, there is little literature on PSA channel estimation techniques for multiple transmitter systems. In [16], an alternating PSA channel estimation scheme was suggested for multiple transmitter systems. To estimate the channel from the $m$ th transmitter to the receiver, the pilot symbols are transmitted only from the $m$ th transmitter, while all the other transmitters either transmit null symbols or stop transmission. With this alternating pilot symbol scheme, $M$ times as many pilot symbols are needed to estimate all the channels in an $M$ transmit antenna system as compared to that required for a single transmit antenna system. The expansion in pilot symbols is undesirable from the standpoint of data throughput and bandwidth efficiency. Here, we propose a multirate PSA channel estimation technique that does not require expansion in the number of pilot symbols for multiple transmitter OFDM systems.

Although the different signals from multiple transmitters in a transmitter diversity system tend to interfere with each other, pilot symbols can be constructed for multiple transmitter OFDM systems to avoid this form of interference and, thus, simplify the task of channel estimation during the pilot mode. Notice in (1) that, for properly designed OFDM transmitter diversity systems, the subchannels for the signal from each transmitter are decoupled, that is, $\boldsymbol{\Lambda}_{1}(n)$ and $\boldsymbol{\Lambda}_{2}(n)$ are diagonal matrices. Therefore, if the pilot symbols are constructed so that pilot symbols transmitted from different transmitters occupy different frequency bins, any individual symbol in the demodulated signal vector $\mathbf{Y}(n)$ will then contain a contribution from only one transmitter, and the complex channel gain for that particular subcarrier can be easily estimated. An obvious choice is to have the pilot symbols among the transmitters evenly distributed while nonoverlapping in frequency. In theory, any pilot symbols that satisfy the nonoverlapping conditions will be sufficient. In practice, the pilot symbols should be chosen to have other desirable OFDM properties as well. Chirp sequences are attractive for channel estimation in OFDM systems because they have a flat power spectrum and a low peak-to-average power ratio [17]. Here, we propose the use of chirp sequences, with different phase offsets from antenna to antenna, as pilot symbols for multiple transmitter OFDM systems. Define a length $K$ chirp sequence as

$$
C(k)=e^{j \pi k^{2} / K}, \quad 0 \leq k \leq K-1 .
$$

Let $P S_{m}(n, k)$ denote the $k$ th tone of the pilot symbol transmitted from the $m$ th transmit antenna during the block instant $n$. The pilot symbols are constructed as

$$
\begin{aligned}
& P S_{m}(n, k+m-1) \\
& \quad= \begin{cases}(-1)^{m} \sqrt{M} C(k+m-1), & \text { if }(k)_{M}=0, \\
0, & \text { otherwise, }\end{cases}
\end{aligned}
$$




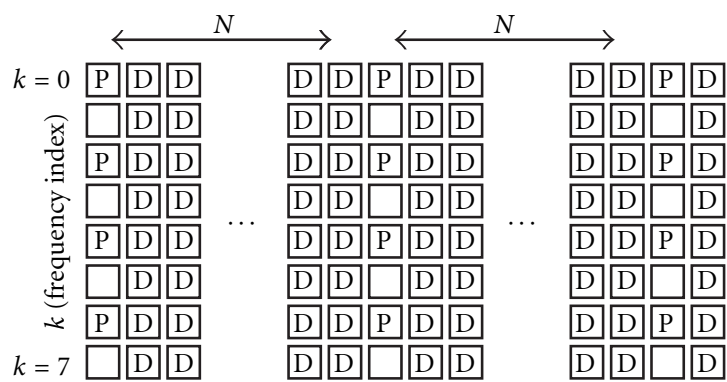

$P S_{1}$
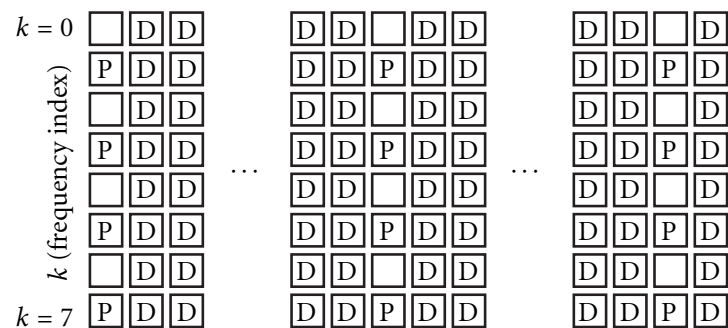

$\mathrm{PS}_{2}$

$n$ (time index)

Legend: $\mathrm{D}$ Data symbol $\quad \mathrm{P}$ Pilot symbol $\square$ Null symbol

FIgURe 2: Pilot symbol patterns for an example OFDM transmitter diversity system with $K=8$ and $M=2$.

where $M$ is the number of transmitters, $(k)_{M}$ denotes $k$ modulo $M, 1 \leq m \leq M, 0 \leq k \leq K-1$, and $1 \leq m+k \leq K$. Figure 2 shows the pilot symbol patterns for an example two-branch OFDM transmitter diversity system.

Since the pilot symbols are known to the receiver and, during the pilot instants, each symbol in $\mathbf{Y}(n)$ contains only the contribution from one transmitter, the least-square estimate $^{3}$ for the $(k+m-1)$ th diagonal element of $\boldsymbol{\Lambda}_{m}(n)$, that is, the complex gain of the $(k+m-1)$ th subcarrier from the $m$ transmitter, is given by

$$
\tilde{\Lambda}_{m}(n, k+m-1)= \begin{cases}\frac{Y(n, k+m-1)}{P S_{m}(n, k+m-1)}, & \text { if }(k)_{M}=0, \\ 0, & \text { otherwise. }\end{cases}
$$

Notice that the nonzero estimate

$$
\tilde{\Lambda}_{m}(n, k+m-1)=\Lambda_{m}(n, k+m-1)+W(n, k+m-1),
$$

where $\Lambda_{m}(n, k+m-1)$ is the actual complex gain of the $(k+$ $m-1)$ th subcarrier from the $m$ th transmitter and $W(n, k+$ $m-1)$ is a zero-mean complex Gaussian random variable with variance $\sigma_{W}^{2}=\sigma_{Z}^{2} / M$.

\footnotetext{
${ }^{3}$ For OFDM systems with fully decoupled subchannels, the least-square channel estimator is equivalent to the zero-forcing channel estimator [5].
}

A number of two-dimensional (2D) filtering techniques have been proposed for PSA channel estimation for OFDM systems. The 2D Wiener filter proposed in $[6,7]$ has fairly high complexity and requires knowledge of the channel statistics. In [11], a robust MMSE interpolator that does not require knowledge of channel statistics was proposed. The interpolator in [11], however, requires 2D filtering, a 2D DFT, and a 2D IDFT. Here, we consider a robust yet simple interpolation approach that does not depend on channel statistics and requires only a simple windowing function and onedimensional interpolation filters.

The diagonal elements of $\tilde{\Lambda}_{m}(n)$ are, in effect, samples of the frequency response of the channel between the $m$ th transmitter and the receiver. Let $\tilde{\mathbf{h}}_{m}(n)$ be the IDFT of the diagonal of $\tilde{\boldsymbol{\Lambda}}_{m}(n)$. In the absence of noise, $\tilde{\mathbf{h}}_{m}(n)$ is related to the actual channel impulse response (CIR) $\mathbf{h}_{m}(n)$ by [18]

$$
\tilde{h}_{m}(n, k)=\frac{1}{M} \sum_{l=0}^{M-1} h_{m}\left(n,\left(k+\frac{K}{M} l\right)_{K}\right) e^{j(2 \pi m / M) l} .
$$

Notice that $\tilde{\mathbf{h}}_{m}(n)$ is the sum of circularly shifted images of $\mathbf{h}_{m}(n)$. The images in (7) are the direct result of sampling in the frequency domain. To avoid aliasing in the time domain, the condition $K \geq M(L+1)$ must be satisfied. To remove the images, $\tilde{\mathbf{h}}_{m}(n)$ is passed through a length $L+1$ rectangular window of gain $M$ to yield the temporal estimate $\hat{\mathbf{h}}_{m}(n)$ at the pilot instant as

$$
\hat{h}_{m}(n, k)= \begin{cases}h_{m}(n, k)+\xi(n, k), & 0 \leq k \leq L \\ 0, & L+1 \leq k \leq K-1 .\end{cases}
$$

The DFT of $\hat{\mathbf{h}}_{m}(n)$ yields the estimate of the channel parameters

$$
\hat{\boldsymbol{\Lambda}}_{m}(n)=\boldsymbol{\Lambda}_{m}(n)+\boldsymbol{\Xi}(n),
$$

where the elements of the noise vector $\boldsymbol{\Xi}(n)$ have a variance of $\sigma_{W}^{2} M(L+1) / K$. Since $M(L+1)<K$ in general, in addition to removing the images, the windowing operation also reduces the variance of the noise by a factor of $M(L+1) / K$. These temporal estimates at the pilot instants, $\hat{\mathbf{h}}_{m}(n)$, are then passed through an interpolation filter to provide the estimated channel parameters during the data transmission mode.

\subsection{Interpolation of channel parameters for multiple transmitter OFDM systems}

Instead of explicitly tracking the multiple time-varying CIRs using a continuous decision-directed algorithm that would require the MMSE solution as in [12], we propose to track the multiple CIRs by interpolating a set of estimated channel responses $\hat{\mathbf{h}}_{m}(n)$ obtained from periodically transmitted pilot symbols $\mathbf{P S}_{m}(n)$, that is, the insertion of one pilot symbol every $N$ transmitted OFDM symbols (see Figure 2). The interpolator takes $Q$ consecutive channel estimates obtained from the pilot symbols at a rate of $1 /\left(N(K+G) T_{S}\right)$, interpolates the estimates with a real-valued finite impulse response 
(FIR) digital filter, and generates $N-1$ interpolated CIR samples at the OFDM symbol rate of $1 /\left((K+G) T_{S}\right)$. These interpolated values provide a robust estimate of the CIRs for the diversity decoder during the data transmission mode.

Notice that the multipath fading process is bandlimited to the maximum Doppler shift frequency $f_{D}$. Therefore, to satisfy the Nyquist criteria, the sampling rate of the channel estimates must satisfy $f_{s}>2 f_{D}$, where the sampling frequency $f_{s}=1 /\left(N(K+G) T_{S}\right)$. The equivalent condition

$$
N<\frac{1}{2 f_{D}(K+G) T_{S}}
$$

gives an upper bound on the pilot symbol spacing. It is well known that the impulse response of the ideal interpolator for bandlimited signals is the sinc function, which has an infinite number of coefficients and is, therefore, unrealizable. A number of practical interpolators have been proposed in $[14,19,20]$. As shown in [19], even order interpolation filters, that is, when $Q$ is odd, do not have linear phase. Nonlinear phase distortion can cause discontinuities in the envelope of the interpolated signal. Furthermore, linear phase interpolation filters have symmetrical coefficients, which can reduce the number of calculations by a factor of 2 . Therefore, we will focus on odd order, linear phase interpolation filters.

In general, the interpolation process improves with increased sampling rates and with higher order interpolation filters. However, there is no analytical expression for the interpolation error of bandlimited signals using these interpolators. Therefore, the interpolation errors of a number of interpolators were simulated to provide a qualitative measure of how well they may track a frequency-selective fading channel. The interpolation performance criteria used is the MSE between the interpolated and the actual CIR. Assuming that the pilot symbols are transmitted at block instants $n=p N$ for $p=0,1,2, \ldots$, the interpolation MSE is defined as

$$
\varepsilon=\frac{1}{N_{T}(N-1) L} \sum_{p=0}^{N_{T}-1} \sum_{n=p N+1}^{p N+N-1} \sum_{l=0}^{L-1}|\hat{h}(n, l)-h(n, l)|^{2},
$$

where $N_{T}$ is the total number of interpolation intervals in the simulation. The interpolation MSEs for an OFDM system with $K=128$ were simulated at $f_{D}=100 \mathrm{~Hz}$ with average received signal-to-noise ratio (SNR) of $40 \mathrm{~dB}$ and at several pilot symbol spacings $N$ to measure the effectiveness of the various interpolation filters at different sampling rates. The COST 207 six-ray typical urban channel power delay profile [21] was used throughout the simulations. Simulation results of the interpolation MSE as a function of the normalized sampling rate, $f_{s} /\left(2 f_{D}\right)$, for the linear interpolator, third and fifth order Lagrange interpolators [19], and third and fifth order least-square interpolators $(\alpha=0.5)$ [20] are shown in Figure 3. Simulation results show that the linear interpolator has significant interpolation error until the sampling rate is well above 4 times the Nyquist rate. As expected, the higher order interpolators all have better performance than the linear interpolator. Although the classical Lagrange interpolation filter is optimally flat in the passband, it has a wider

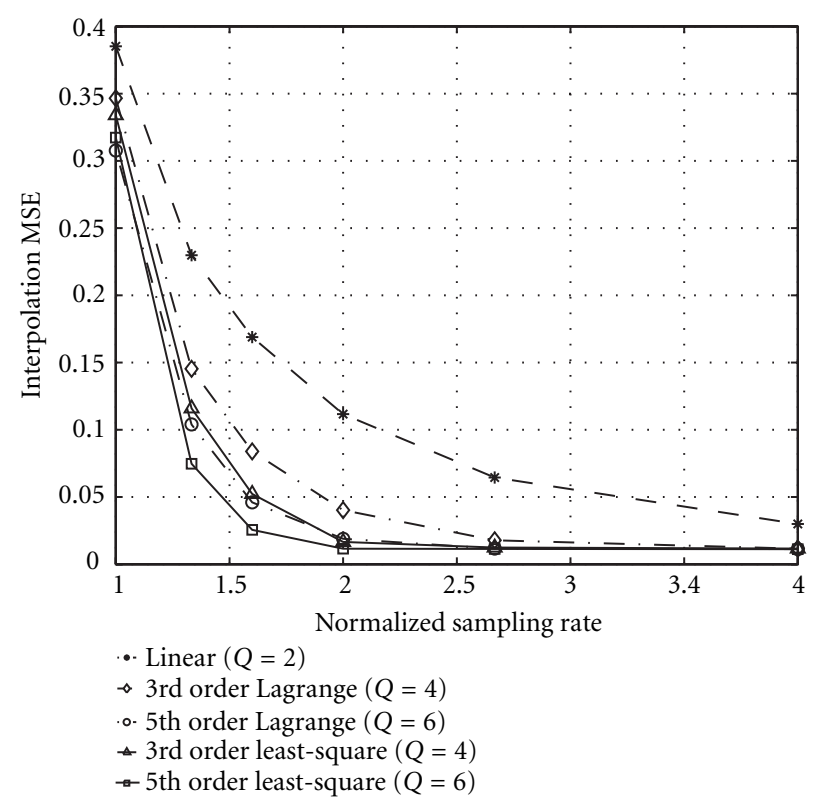

FIGURE 3: Interpolation error as a function of the normalized sampling rate $f_{s} /\left(2 f_{D}\right)$.

transition band and has less stopband rejection than other types of "optimum" filters such as the least-square filter [20]. Figure 3 also shows that the least-square filters have a lower interpolation MSE than the Lagrange interpolation filters of the same order. Therefore, the least-square filter is a better choice for interpolating a bandlimited signal. Interestingly, the interpolation errors of the fifth order Lagrange, and the third and fifth order least-square interpolators are very close to the error floor at only twice the Nyquist rate.

Since the insertion of pilot symbols represents a loss of bandwidth efficiency, a main objective in designing PSA channel estimators is to minimize the sampling rate or the number of pilot symbols, that is, to maximize $N$. Another practical consideration is minimization of the complexity and delay of the interpolator, which usually translates to using the lowest order interpolator possible. From the above simulation results, the third order least-square interpolator operating at about twice the Nyquist rate, that is, $N \approx$ $1 /\left(4 f_{D}(K+G) T_{S}\right)$, should achieve good interpolation performance at a reasonable sampling rate, implementation complexity, and delay. The hardware complexity of the interpolation filter can be further reduced by employing the polyphase filter structure as shown in [20]. A block diagram of the proposed PSA channel estimator for a two-branch OFDM transmitter diversity system is shown in Figure 4.

\subsection{Performance of pilot-symbol-assisted channel estimators}

Channel estimators based on the pilot symbols and interpolators described in Sections 3.1 and 3.2 have been evaluated with the two-branch space-time block-coded OFDM (STBCOFDM) and space-frequency block-coded OFDM (SFBCOFDM) transmitter diversity systems proposed in $[3,4]$. For 

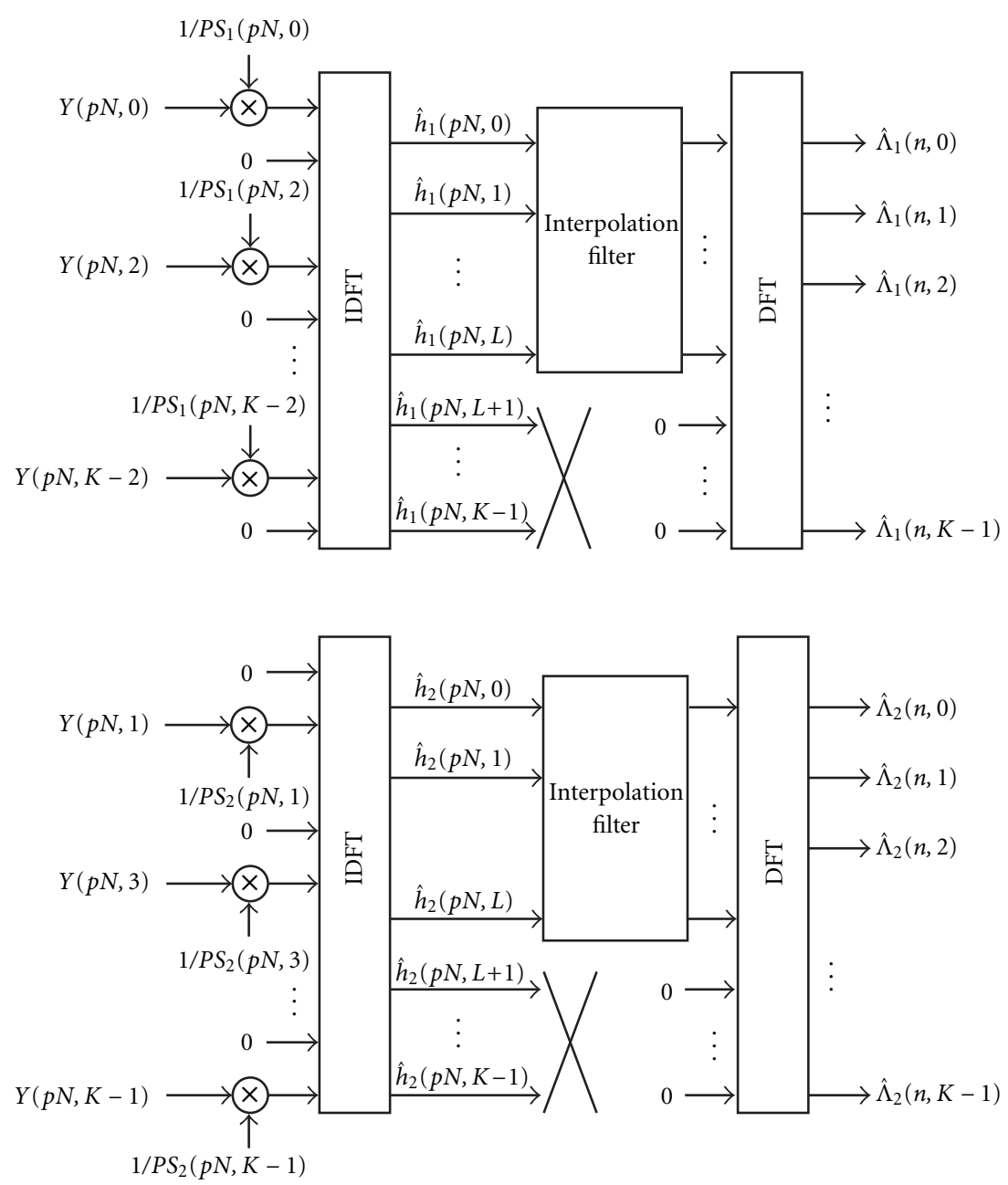

FIGURE 4: Block diagram of the proposed PSA channel estimator for a two-branch OFDM transmitter diversity system.

the STBC-OFDM simulations, the system employed 128 subcarriers with 4-QAM modulation at a symbol rate of $2^{20}$ symbols per second on each subcarrier, that is, $K=128$ and $T_{S}=$ $2^{-20}$ seconds. The pilot symbol spacing was set at $N=20$ so that the sampling frequency was near twice the Nyquist rate at a maximum Doppler frequency of $100 \mathrm{~Hz}$. Simulation results of the average bit error rate (BER) performance for a two-branch STBC-OFDM system with ideal channel parameters and with channel parameters estimated by a third order Lagrange interpolator are shown in Figure 5. Comparisons to a third order least-square interpolator are shown in Figure 6. Simulation results confirm that at slow fading conditions, such as $f_{D}=50 \mathrm{~Hz}$, both the third order Lagrange and third order least-square interpolators perform very well. In fact, for this fading rate there is no noticeable BER degradation between the systems using the ideal channel parameters and those using the estimated parameters from the third order least-square interpolator. At $f_{D}=100 \mathrm{~Hz}$, which corresponds to sampling at about twice the Nyquist rate, the BER performance with the Lagrange interpolator is degraded slightly, while that with the least-square interpolator still shows very little degradation. This relative performance is in agreement with the interpolation MSE results in Figure 3, where the third order least-square interpolator has a lower interpolation MSE than the third order Lagrange interpolator. At a faster fading condition of $f_{D}=150 \mathrm{~Hz}$, which corresponds to sampling at about 1.4 times the Nyquist rate, the BER performances of both systems with estimated channel parameters are severely degraded as a result of the excessively high interpolation MSE.

For the SFBC-OFDM simulations, the system employed 256 subcarriers with 4-QAM modulation at a symbol rate of $2^{20}$ symbols per second on each subcarrier, that is, $K=256$ and $T_{S}=2^{-20}$ seconds. The pilot symbol spacing was set at $N=10$, so that the sampling frequency was again at about twice the Nyquist rate for a maximum Doppler frequency of $100 \mathrm{~Hz}$. Simulation results of the average BER performance for a two-branch SFBC-OFDM system with ideal channel parameters and with channel parameters estimated by a third order Lagrange interpolator are shown in Figure 7. Simulation results with a third order least-square interpolator are shown in Figure 8. Simulation results of the SFBCOFDM system show that at slow fading conditions, such as $f_{D}=50 \mathrm{~Hz}$, both the third order Lagrange and third 


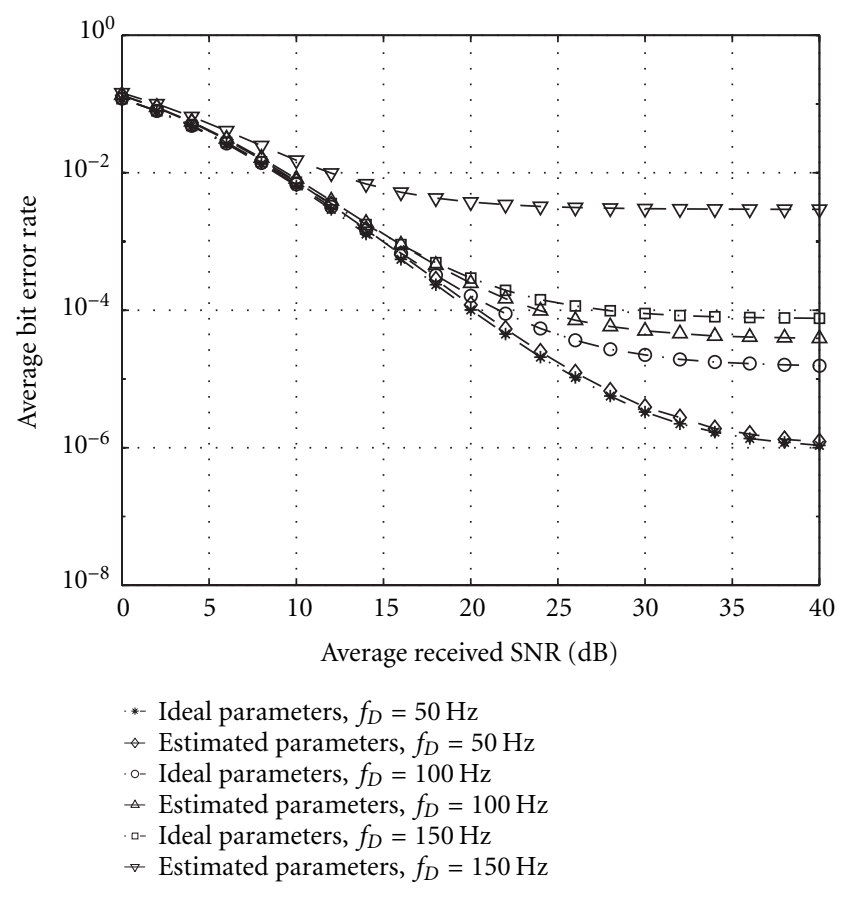

FIgURE 5: Performance comparison of STBC-OFDM systems with ideal channel parameters and channel parameters estimated by a third order Lagrange interpolator.

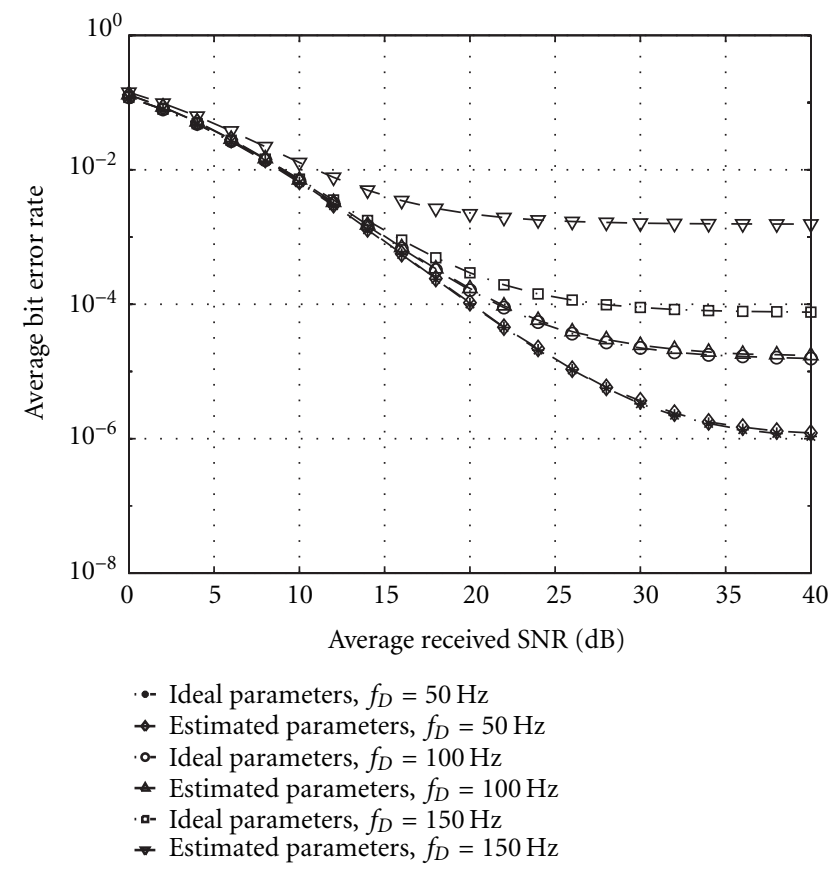

FIgURe 6: Performance comparison of STBC-OFDM systems with ideal channel parameters and channel parameters estimated by a third order least-square interpolator.

order least-square interpolators have similar performance, and there is only a slight degradation for the systems using the estimated parameters compared to the systems with

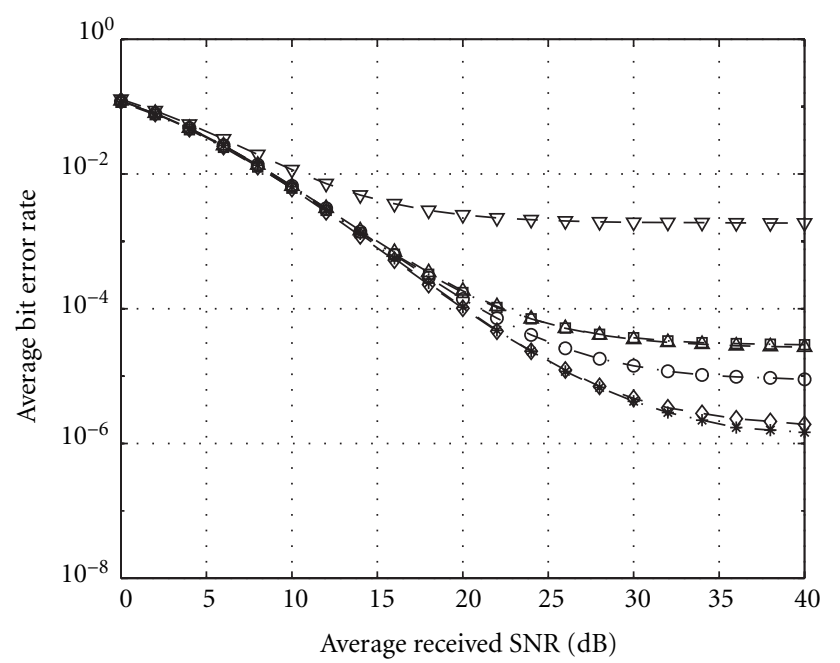

*- Ideal parameters, $f_{D}=50 \mathrm{~Hz}$

$\diamond$ Estimated parameters, $f_{D}=50 \mathrm{~Hz}$

- - Ideal parameters, $f_{D}=100 \mathrm{~Hz}$

$\triangle$ Estimated parameters, $f_{D}=100 \mathrm{~Hz}$

- - Ideal parameters, $f_{D}=150 \mathrm{~Hz}$

* Estimated parameters, $f_{D}=150 \mathrm{~Hz}$

Figure 7: Performance comparison of SFBC-OFDM systems with ideal channel parameters and channel parameters estimated by a third order Lagrange interpolator.

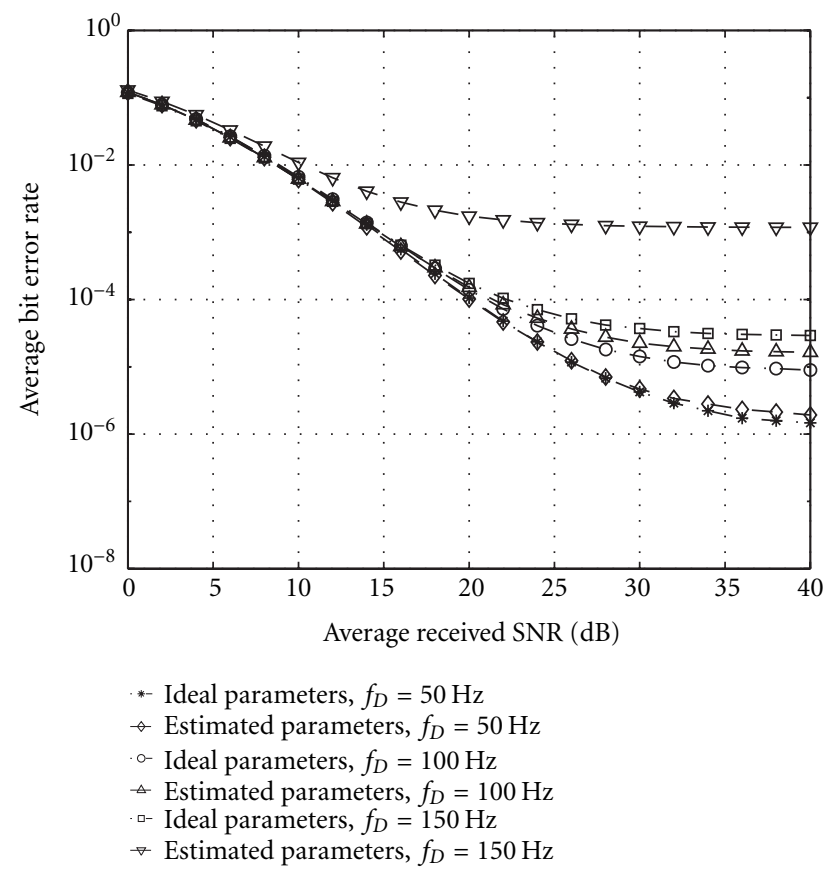

Figure 8: Performance comparison of SFBC-OFDM systems with ideal channel parameters and channel parameters estimated by a third order least-square interpolator.

the ideal channel parameters. At $f_{D}=100 \mathrm{~Hz}$, which corresponds to sampling at about twice the Nyquist rate, the BER performance for the least-square interpolator is clearly 
better than that of the Lagrange interpolator. At a faster fading condition of $f_{D}=150 \mathrm{~Hz}$, which corresponds to sampling at about 1.4 times the Nyquist rate, the BER performances of both interpolators are severely degraded. Clearly, a sufficient sampling rate is crucial to the performance of the proposed PSA channel estimator. From the above simulation results and the earlier interpolation MSE analysis, a good rule-of-thumb is to set the pilot symbol spacing at about twice the Nyquist rate for the anticipated maximum Doppler frequency.

The simulation results for the SFBC-OFDM are generally similar to that of the STBC-OFDM system shown previously in Figures 5 and 6, where the third order least-square interpolator slightly outperforms the third order Lagrange interpolator and both interpolators perform reasonably well when the channel fading rate is at or below the anticipated maximum Doppler frequency.

\subsection{Comparison with the decision-directed MMSE channel estimator}

In this section, the PSA channel estimator proposed in Section 3.2 is briefly compared to the decision-directed MMSE channel estimator of [12]. ${ }^{4}$ As mentioned previously in Section 3, that decision-directed channel estimator is susceptible to error propagation. Therefore, the performance of the decision-directed channel estimator depends on the number of errors in the decisions or decoded symbols used to direct the channel estimates. To improve the performance of the decision-directed channel estimator, the decoded symbols after error-correction decoding are often used for updating the channel estimation. Hence, the performance of the decision-directed channel estimator is affected by the performance of the particular error-correction code employed by the system. Here, instead of arbitrarily choosing an error-correction coding scheme, a lower bound for the BER of the decision-directed MMSE channel estimator was simulated by using the actual symbols in directing the channel estimation. The simulations used the same STBC-OFDM and SFBC-OFDM system parameters as the systems simulated in Section 3.3. The training symbols in [12] were used for the decision-directed MMSE channel estimator and the training symbols were sent at the same spacing as the pilot symbols for the corresponding PSA channel estimator. Figure 9 shows the simulation results comparing the decision-directed MMSE channel estimator with the PSA channel estimator using a third order least-square interpolation filter for the STBC-OFDM system. Figure 10 shows the same comparison for the SFBC-OFDM system. It is interesting to note that, for these particular STBC-OFDM and SFBC-OFDM systems, the PSA channel estimator significantly outperforms the decision-directed MMSE channel estimator under all fading conditions. These results further

${ }^{4}$ In [12], a simplified approach was proposed that required identification of the significant taps of $\mathbf{h}_{m}(n)$. Here, we consider only the basic approach for comparison.

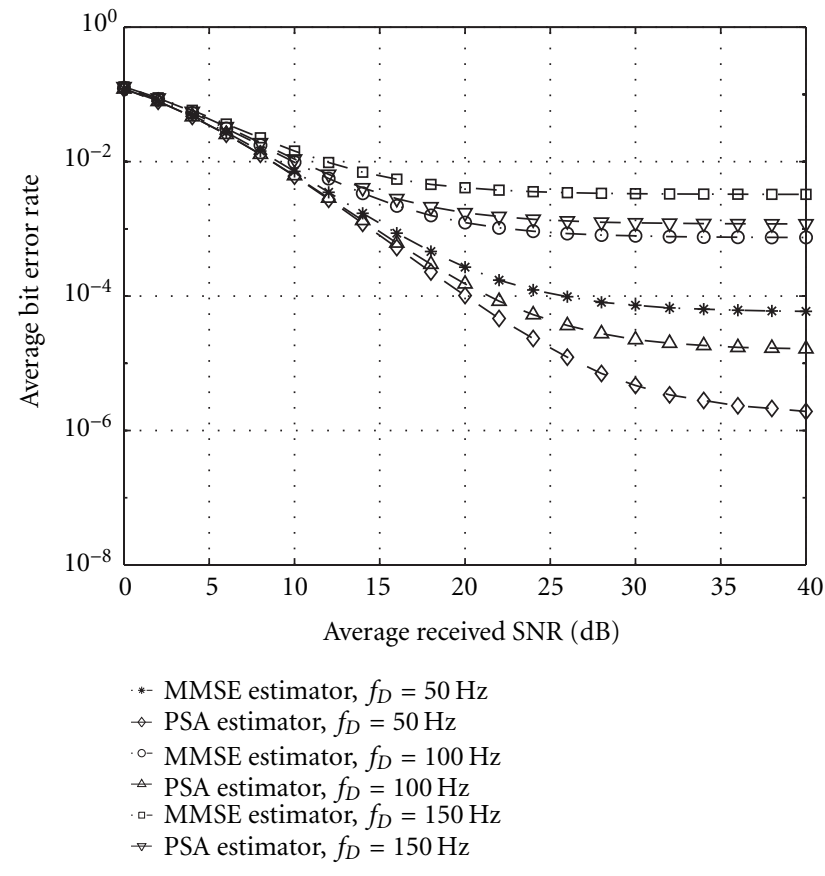

FIGURE 9: Performance comparison of STBC-OFDM systems with channel parameters estimated by a decision-directed MMSE channel estimator and by the PSA channel estimator.

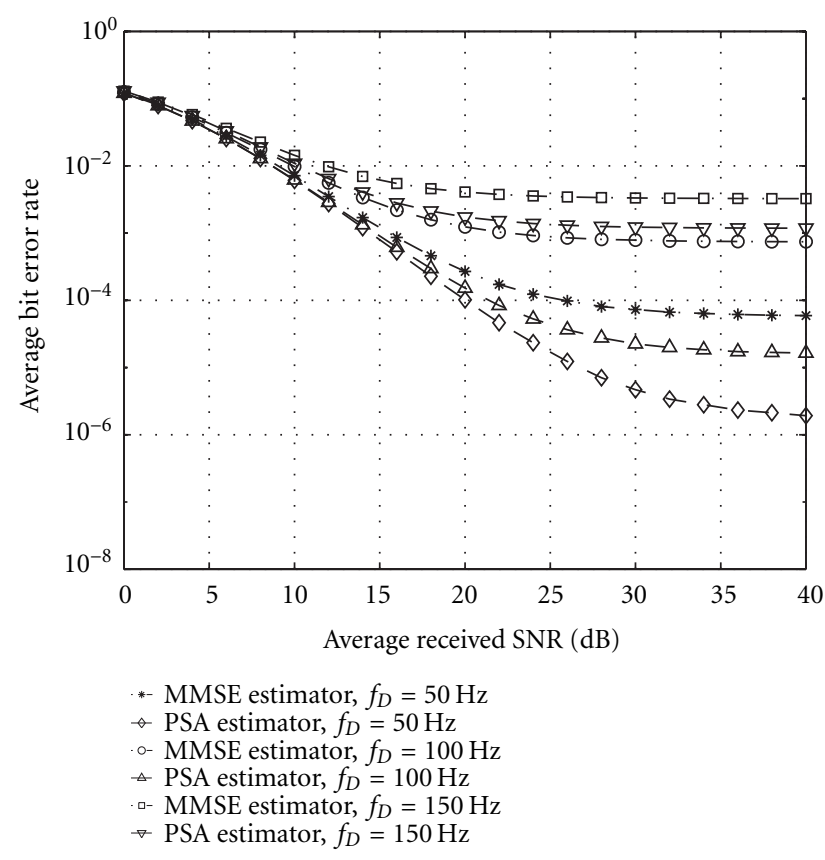

FIGURE 10: Performance comparison of SFBC-OFDM systems with channel parameters estimated by a decision-directed MMSE channel estimator and by the PSA channel estimator.

support the earlier suggestion that the PSA channel estimator is the better choice for OFDM transmitter diversity systems.

The computational complexities of the PSA channel 
TABLE 1: Computational complexities of the PSA channel estimator and the decision-directed MMSE channel estimator.

\begin{tabular}{lcc}
\hline & Multiplications & Additions \\
\hline PSA channel estimator & $((M+M / N) / 2) K \log _{2} K+K / N+M(L+1) Q$ & $(M+M / N) K \log _{2} K+M(L+1)(Q-1)$ \\
MMSE channel estimator & $\left(\left(2 M+M^{2}\right) / 2\right) K \log _{2} K+M K+(M L)^{3} / 3$ & $\left(2 M+M^{2}\right) K \log _{2} K+(M L)^{3} / 3$ \\
\hline
\end{tabular}

*Assuming $K$ is a power of two, each FFT requires $K / 2 \log _{2} K$ multiplications and $K \log _{2} K$ additions [22], and Gaussian elimination with an $n \times n$ matrix requires $n^{3} / 3$ multiplications and $n^{3} / 3$ additions [23].

estimator and the decision-directed MMSE channel estimator are shown in Table 1. Compared to the MMSE channel estimator, the PSA channel estimator requires fewer DFTs: $M+M / N$ for the PSA estimator versus $2 M+M^{2}$ for the MMSE estimator. Furthermore, calculating the MMSE solution for the decision-directed estimator has a complexity of $O\left(M^{3} L^{3}\right)$, while the interpolation filter for the PSA estimator has a complexity that is only proportional to $M L$. Clearly, the PSA channel estimator is computationally more efficient than the decision-directed MMSE channel estimator.

\section{SUMMARY}

A low complexity, bandwidth efficient, pilot-symbol-assisted channel estimator for OFDM transmitter diversity systems has been presented. Different interpolation algorithms have been evaluated and were seen to provide robust channel parameter estimates in various fading environments. Simulation results verify that the proposed technique is well suited for channel estimation in space-time coded OFDM transmitter diversity systems. It has also been shown that the proposed PSA channel estimator outperforms the decisiondirected MMSE channel estimator and is also more computationally efficient.

For ease of presentation, this paper has focused on systems with multiple transmit antennas and a single receive antenna. It should be noted that the proposed approach can also be applied to systems with multiple receive antennas by replicating the proposed channel estimator for each receive antenna.

\section{ACKNOWLEDGMENT}

This paper was presented in part at the IEEE International Conference on Acoustics, Speech, and Signal Processing (ICASSP), Salt Lake City, Utah, USA, May 2001.

\section{REFERENCES}

[1] D. Agrawal, V. Tarokh, A. Naguib, and N. Seshadri, "Spacetime coded OFDM for high data-rate wireless communication over wideband channels," in Proc. IEEE Vehicular Technology Conference, vol. 3, pp. 2232-2236, Ottawa, Ont., Canada, May 1998.

[2] Y. Li, J. C. Chuang, and N. R. Sollenberger, "Transmitter diversity for OFDM systems and its impact on high-rate data wireless networks," IEEE Journal on Selected Areas in Communications, vol. 17, no. 87, pp. 1233-1243, 1999.
[3] K. F. Lee and D. B. Williams, "A space-time coded transmitter diversity technique for frequency selective fading channels," in Proc. IEEE Sensor Array and Multichannel Signal Processing Workshop, pp. 149-152, Cambridge, Mass, USA, March 2000.

[4] K. F. Lee and D. B. Williams, "A space-frequency transmitter diversity technique for OFDM systems," in Proc. IEEE Global Telecommunications Conference, vol. 3, pp. 1473-1477, San Francisco, Calif, USA, November 2000.

[5] J.-J. van de Beek, O. Edfors, M. Sandell, S. K. Wilson, and P. O. Börjesson, "On channel estimation in OFDM systems," in Proc. IEEE Vehicular Technology Conference, vol. 2, pp. 815819, Chicago, Ill, USA, July 1995.

[6] P. Hoeher, S. Kaiser, and P. Robertson, "Two-dimensional pilot-symbol-aided channel estimation by wiener filtering," in Proc. IEEE Int. Conf. Acoustics, Speech, Signal Processing, vol. 3, pp. 1845-1848, Munich, Germany, April 1995.

[7] P. Hoeher, S. Kaiser, and P. Robertson, "Pilot-symbol-aided channel estimation in time and frequency," in Proc. IEEE GLOBECOM Communication Theory Mini-Conference, vol. 3, pp. 90-96, Phoenix, Ariz, USA, November 1997.

[8] O. Edfors, M. Sandell, J.-J. van de Beek, S. K. Wilson, and P. O. Börjesson, "OFDM channel estimation by singular value decomposition," IEEE Trans. Communications, vol. 46, no. 7, pp. 931-939, 1998.

[9] O. Edfors, M. Sandell, J.-J. van de Beek, S. K. Wilson, and P. O. Börjesson, "Analysis of DFT-based channel estimators for OFDM," Wireless Personal Communications, vol. 12, no. 1, pp. 55-70, 2000.

[10] Y. Li, L. J. Cimini Jr., and N. R. Sollenberger, "Robust channel estimation for OFDM systems with rapid dispersive fading channels," IEEE Trans. Communications, vol. 46, no. 7, pp. 902-915, 1998.

[11] Y. Li, "Pilot-symbol-aided channel estimation for OFDM in wireless systems," IEEE Transactions on Vehicular Technology, vol. 49, no. 4, pp. 1207-1215, 2000.

[12] Y. Li, N. Seshadri, and S. Ariyavisitakul, "Channel estimation for OFDM systems with transmitter diversity in mobile wireless channels," IEEE Journal on Selected Areas in Communications, vol. 17, no. 3, pp. 461-471, 1999.

[13] Y. Li, "Optimum training sequences for OFDM systems with multiple transmit antennas," in Proc. IEEE Global Telecommunications Conference, vol. 3, pp. 1478-1482, San Francisco, Calif, USA, November 2000.

[14] S. Sampei and T. Sunaga, "Rayleigh fading compensation for QAM in land mobile radio communications," IEEE Transactions on Vehicular Technology, vol. 42, no. 2, pp. 137-47, 1993.

[15] J. K. Cavers, "An analysis of pilot symbol assisted modulation for Rayleigh fading channels," IEEE Transactions on Vehicular Technology, vol. 40, no. 4, pp. 686-693, 1991.

[16] S. M. Alamouti, "A simple transmitter diversity scheme for wireless communications," IEEE Journal on Selected Areas in Communications, vol. 16, no. 8, pp. 1451-1458, 1998.

[17] J. M. Cioffi and J. A. C. Bingham, "A data-driven multitone 
echo canceller," IEEE Trans. Communications, vol. 42, no. 10, pp. 2853-2869, 1994.

[18] N. J. Fliege, Multirate digital signal processing : multirate systems, filter banks, wavelets, Wiley, Chichester, NY, USA, 1994.

[19] R. W. Schafer and L. R. Rabiner, "A digital signal processing approach to interpolation," Proceedings of the IEEE, vol. 61, no. 6, pp. 692-702, 1973.

[20] G. Oetken, T. W. Parks, and H. W. Schussler, "New results in the design of digital interpolators," IEEE Trans. Acoustics, Speech, and Signal Processing, vol. 23, no. 3, pp. 301-309, 1975.

[21] Commission of the European Communities, Digital Land Mobile Radio Communications-COST 207, Office for Official Publications of the European Communities. Luxembourg, 1989.

[22] A. V. Oppenheim and R. W. Schafer, Discrete-time signal processing, Prentice Hall, Englewood Cliffs, NJ, USA, 1989.

[23] G. H. Golub and C. F. Van Loan, Matrix computations, Johns Hopkins University Press, Baltimore, Md, USA, 3rd edition, 1996.

King F. Lee received his BSEE from the University of Florida, the MSE from Florida Atlantic University, and the Ph.D. degree from the Georgia Institute of Technology. In 1979, he joined Motorola Inc., where he is currently a Distinguished Member of the Technical Staff. His areas of interest include mixed analog-digital integrated circuit design, computer aided circuit design, wireless communications, digital signal and im-

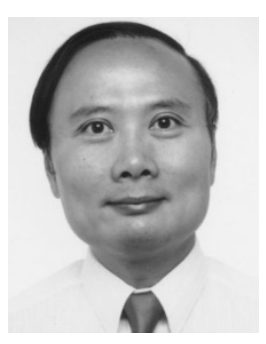
age processing. He has served as a Member of the Industrial Advisory Board of the NSF Research Center for the Design of AnalogDigital Integrated Circuits (CDADIC) from 1989 to 1993 and is a Registered Professional Engineer.

Douglas B. Williams received his BSEE, MS, and Ph.D. degrees in electrical and computer engineering from Rice University, Houston, Texas, in 1984, 1987, and 1989, respectively. In 1989, he joined the faculty of the School of Electrical and Computer Engineering at the Georgia Institute of Technology, Atlanta, Georgia, where he is currently an Associate Professor. There he is also affiliated with the Center for Signal and

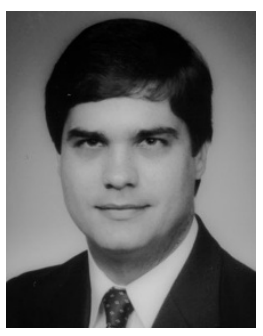
Image Processing and teaches courses in signal processing and telecommunications. Dr. Williams has served as an Associate Editor of the IEEE Transactions on Signal Processing and is a member of the IEEE Signal Processing Society's SPTM Technical Committee. He was on the conference committee for the 1996 International Conference on Acoustics, Speech, and Signal Processing that was held in Atlanta and is currently co-chair of the 2002 IEEE DSP and Signal Processing Education workshops. Dr. Williams was coeditor of the Digital Signal Processing Handbook published in 1998 by CRC Press and IEEE Press. He is a member of the Tau Beta Pi, Eta Kappa Nu, and Phi Beta Kappa honor societies. 